\title{
Alocação de pedidos em aglomerados industriais calçadistas: modelos e estudo de caso
}

\author{
Gustavo Beltran de Souza \\ Univ. Federal de São Carlos - UFSCar \\ Flávio César Faria Fernandes \\ Univ. Federal de São Carlos - UFSCar
}

\section{Resumo}

Este artigo tem como objetivo apresentar propostas de solução (modelos matemáticos) para a alocação de pedidos em aglomerados industriais calçadistas. Definimos, como base para a sustentação de tais propostas, uma classificação dos aglomerados industriais calçadistas e a definição de uma infra-estrutura tecnológica coordenada por um agente central do cluster. 0 caso de um cluster exportador também é apresentado.

\section{Palavras-chave}

Aglomerado Industrial, Planejamento e Controle da Produção, Sistemas de Apoio à Decisão, Sistemas de Informação, Programação Inteira 0-1.

\section{Allocation of orders in footwear industrial clusters: models and case study}

\footnotetext{
Abstract

This paper aims to show proposals of solution (mathematical models] for the allocation of orders in footwear industrial clusters. We define, as base for the supporting of this proposal, a classification of footwear industrial clusters and the definition of a technological infrastructure coordinated by a cluster's central agent. The case of an exporter cluster is also presented.

Key words

Production Planning and Control, Industrial Cluster, Decision Support System, Information System, Integer Programming 0-1.
} 


\section{INTRODUĈ̣̃O}

O presente artigo combina de forma inédita dois temas ainda não tratados na literatura de forma conjunta, a saber: a) a organização da produção por meio de aglomerados industriais (Industrial Clusters) e b) a alocação de pedidos, que é um problema da função Planejamento e Controle da Produção (PCP), por meio de modelos matemáticos. Assim, primeiramente é feita uma breve revisão introdutória (seção 1) relativa a aglomerados industriais e PCP de modo a caracterizar um pano de fundo para a definição do problema a ser estudado (seção 2). Na seção 3 são apresentados os modelos de solução do problema tratado. A seção 4 apresenta um estudo de caso no ramo calçadista e a seção 5 encerra o artigo com as considerações finais e conclusões.

O contexto empresarial está atualmente caracterizado pela busca constante por competitividade, ou seja, empresas de diversos ramos buscam estratégias e formas de atuação mais efetivas para conquistar determinados mercados. É neste contexto que vários autores (PORTER, 1990, 1998; AMATO NETO, 1999; SANTOS et al., 1994; COUTINHO \& FERRAZ, 1994; McCORMICK, 1999; SCHMITZ, 1992; RABELLOTTI, 1995, 1999 e CAMAGNI, 1991) têm destacado a importância dos aglomerados industriais (Industrial Clusters) para o aumento da competitividade principalmente das pequenas e médias empresas.

Um Industrial Cluster pode ser definido como aglomeração de empresas de um mesmo ramo que são encontradas geograficamente próximas umas das outras, mantendo suas relações por meio do desenvolvimento e uso de tecnologias para sistemas de produção comuns, compartilhando tecnologia, conhecimento (experiências), informação, mão-de-obra especializada, fornecedores, clientes, etc., isto é, sua principal finalidade é unir esforços para alcançar objetivos também considerados comuns. Aglomerados industriais possuem grande poder de inovação, tanto em aspectos tecnológicos quanto organizacionais, fato que os torna importantes em relação à atuação de tais indústrias num mercado competitivo, aumentando a produtividade e estimulando a formação de novos negócios para seus membros, podendo ter grande escala de produção sem sacrificar a flexibilidade (RABELLOTTI, 1993; SCHMITZ, 1995).

Para Schmitz (1999), vários autores enfatizam a importância da proximidade geográfica nas relações interfirmas. Termos como sinergia, economias de aglomeração, competitividade sistêmica, sistemas de inova- ção locais ou eficiência coletiva expressam os principais assuntos sobre esta questão. Um dos principais resultados da pesquisa de Schmitz (1999) realizada no período de 1992 a 1997 em empresas exportadoras de calçados do Vale dos Sinos (Sul do Brasil) consiste na positiva e significante relação entre cooperação e desempenho, de modo que as empresas que aumentaram a cooperação progrediram mais do que aquelas que não aumentaram. No entanto, verificou-se que o desempenho dessas empresas variava com o tipo de cooperação adotado.

Diante das condições proporcionadas pelos aglomerados industriais, vale a pena destacar que o objetivo deste artigo é apresentar soluções que viabilizem tais sinergias, isto é, apresentar soluções para a produção cooperada (distribuída) de aglomerados industriais calçadistas. Com a produção cooperada, torna-se possível o aumento da escala de produção e a exportação de calçados, algo que de forma isolada as pequenas empresas do cluster não conseguiriam. Vale destacar que a alocação de pedidos às fábricas envolvidas (pertencentes ao cluster) é o principal processo de decisão destes ambientes.

É importante considerar que a busca por vantagens competitivas, baseando-se no conceito de eficiência coletiva, tem como fator decisivo a confiança entre as relações de cooperação das empresas. Muitas vezes tal confiança só é obtida quando existe um certo equilíbrio entre a cooperação e a competição das empresas que se relacionam.

\section{A alocação de pedidos às fábricas de um - cluster é o principal processo de decisão em ambientes de produção distribuídos.}

Conforme Day et al. (2000), a confiança e a cooperação entre as empresas de um Industrial Cluster são centrais para sua existência. Para que as empresas estabeleçam um ambiente criativo e inovador elas devem compartilhar suas habilidades e informações com as demais. Esta mistura de confiança e dependência só pode ser consolidada com a criação de uma infra-estrutura de PCP baseada em procedimentos inteligíveis e considerados justos.

Certamente, cooperações formais ou informais trarão ganhos de competitividade, entretanto, cooperação no sentido de comprometimento (formalismo) faz com que as empresas de um cluster reflitam sobre o ato de coordenar esta aliança. É justamente com essa preocupação que a busca por soluções baseadas em recursos como 
as redes de computadores e as tecnologias de comunicação (Sistemas de Informação/Sistemas de Apoio à Decisão) podem tornar factíveis aos grupos de empresas a coordenação de suas atividades comuns, aumentando a capacidade de processar informações e permitindo que as empresas possam oferecer produtos customizados mantendo grande volume de produção. Vale frisar que o aumento da variedade de produtos tem tremendo impacto sobre a complexidade da integração da informação tratada pelos Sistemas de Planejamento e Controle da Produção (SPCP).

Características como respostas rápidas às necessidades dos clientes e às mudanças imprevisíveis nas condições de produção são altamente dependentes de sistemas de planejamento flexíveis e de mecanismos de controle confiáveis (SOARES et al., 2000; FERNANDES \& MATTIUZZO, 1986; CORRÊA \& GIANESI, 1997). distribuídos em Sikora \& Shaw (1998), Kovacs \& Mezgar (1998), Azevedo \& Sousa (2000) e Mezgar et al. (2000).

\section{O PROBLEMA ESTUDADO}

Para que se possa alcançar o objetivo destacado na introdução deste artigo (viabilizar a produção cooperada em aglomerados industriais calçadistas), é relevante que se esclareça, mesmo que de uma forma geral, o processo de produção do calçado. Desta forma, os principais processos são: preparação do material, corte, pesponto, montagem e expedição.

A preparação do material tem como objetivo preparar e disponibilizar os materiais descritos na ordem de produção para que os mesmos sejam cortados no processo seguinte. Após o corte, as peças que fazem parte do cabedal são organizadas em lotes e encaminhadas à seção de pesponto, onde são preparadas, chanfradas, dobradas, picotadas, coladas e em seguida costuradas. Na maior parte das empresas, esse trabalho é realizado manualmente e, em algumas poucas, feito em máquinas eletrônicas simples. O cabedal corresponde à parte de cima do pé e divide-se em gáspea (parte da

Já o papel de comando e coordenação do SPCP é evidenciado por Contador \& Contador (1997) ao afirmarem que o SPCP corresponde à medula no corpo humano. Os sistemas de informação atualmente disponíveis não satisfazem as necessidades do planejamento e controle distribuídos, como por exemplo a falta de suporte à produção coordenada dentro de uma rede ou ainda em empresas com processos de negócio amplos, além disso, a necessidade da gestão da cadeia de abastecimento implica em mudanças fundamentais no projeto de sistemas de informação para o planejamento e controle (VOLLMANN et al., 1997).

O planejamento, a programação e o controle dos fluxos de pedidos gerenciados de forma ampla pela empresa coordenadora correspondem ao grande desafio de organizações com processos de produção distribuídos (RICHARDS et al., 1997; ZHOU \& BESANT, 1999). Nesses trabalhos são listadas em comum algumas funcionalidades de um sistema distribuído (solução X-CITTIC) para as operações relacionadas às necessidades da indústria de semicondutores.

Conforme Lecompte et al. (2000), para um ambiente de produção distribuído, além da necessidade de possuir um sistema de planejamento e controle da produção há também a preocupação em se definir agentes (elementos) capazes de sustentar a coordenação deste ambiente. Veja mais detalhes sobre ambientes de produção frente), traseiro e lateral. Normalmente, é constituído de várias peças e reforços, usados para dar mais firmeza e proteção à parte superior do pé ou, então, por questão de design. A montagem basicamente une o salto, a sola e o cabedal (ANDRADE \& CORRÊA, 2001).

Obter uma produção cooperada efetiva implica em identificar e analisar critérios como a capacidade produtiva disponível de cada fábrica do cluster (na maioria das vezes variando conforme o produto solicitado), o prazo de término das operações produtivas em função do tamanho do lote distribuído, a qualidade das operações e dos produtos e principalmente, o cumprimento dos prazos prometidos ao cliente. A identificação e análise dos critérios citados acima compõem o problema tratado neste artigo, ou seja, compõem o processo decisório que envolve a alocação de pedidos em aglomerados industriais calçadistas.

No entanto, outro fator relevante para a concepção das propostas de solução que envolve a alocação de pedidos é a identificação das operações produtivas de cada fábrica do cluster, isto é, para cada fábrica do cluster deve-se identificar quais as operações relacionadas à produção do calçado (corte, pesponto e montagem) são realizadas. Tal identificação nos permite classificar os aglomerados industriais calçadistas em três tipos.

Neste artigo, um cluster é classificado como Cluster Multiprocesso se todas as fábricas realizam todos os 
processos de produção. Cluster Monoprocesso é aquele em que cada fábrica realiza um único processo de produção (corte, pesponto ou montagem); tal tipo é característico do modelo italiano de cluster. Por fim, o terceiro tipo compreende os clusters onde os dois tipos anteriores de fábricas são encontradas (fábricas multiprocesso e fábricas monoprocesso), o que os classifica como Clusters Híbridos. Os mecanismos de alocação de pedidos dependem da classe do Industrial Cluster. Esta relação de dependência é apresentada na Tabela 1.

A implementação de qualquer uma destas propostas de solução (mecanismos de alocação) exige a elaboração de uma infra-estrutura de coordenação para que a alocação de pedidos ocorra da melhor maneira possível. Frente a esta questão, um agente coordenador para o cluster deverá ser criado e atribuída a ele toda a responsabilidade da alocação dos pedidos, ou seja, por meio das informações contidas em uma base de dados central, ele analisa os critérios e as restrições de alocação (capacidade disponível do recurso produtivo, prazo máximo de término da operação, tipo de produto, fábricas capazes de produzir com qualidade o pedido, etc.) que fazem parte e que são essenciais para ambientes de produção distribuídos. A obtenção das informações também é responsabilidade do agente coordenador, isto é, as informações essenciais de cada fábrica devem ser extraídas de suas respectivas bases de dados pelo coordenador e armazenadas na Base de Dados Central.

Esta operação de obtenção dos dados ocorrerá por meio de Interfaces de Comunicação (IC) padronizadas, ou seja, a informação solicitada pelo coordenador deverá ser disponibilizada pelos SPCP das fábricas com a formatação dos dados estabelecida pelo agente solicitante. Cada fábrica deve, ao mesmo tempo, conservar as informações de suas base de dados sempre atualizadas e consistentes para que a tomada de decisão da coordenadora seja confiável e efetiva. Para que o sistema de alocação seja efetivo, é imprescindível que cada fábrica do cluster tenha um bom sistema de apontamento / monitoramento / acompanhamento da produção a ser ainda realizada, já realizada e que está sendo realizada. É exatamente esse sistema que vai garantir que a base de dados da fábrica (B.D. local) sempre esteja atualizada; este tópico não é objeto de estudo e aprofundamento neste artigo.

\section{tipo de cluster define o mecanismo de alocação de pedidos.}

A concepção de Interfaces de Comunicação padronizadas traz como grandes vantagens a flexibilidade na obtenção dos dados de diversos sistemas de informação, independentemente da linguagem, base de dados e plataforma nas quais foram desenvolvidos, além da escalabilidade, que corresponde à capacidade de formar novas parcerias ou desfazer algumas. Outro fator relevante da Interface de Comunicação corresponde também à sua função de integração da informação de um Industrial Cluster. De forma simples e direta, uma Interface de Comunicação corresponde a um arquivo com dimensões padronizadas, isto é, suas seções e campos estão distribuídos de acordo com coordenadas preestabelecidas. Sua função é viabilizar o envio e a leitura dos dados relacionados a uma determinada ocorrência (atualizações e resultados de processos decisórios). A Figura 1 faz uma representação da infra-estrutura de coordenação, considerando a integração da informação com suas interfaces de comunicação (setas tracejadas).

Pode-se notar nesta figura que outro componente essencial para a integração entre o sistema de PCP do coordenador e os sistemas de PCP das fábricas é a Base de Dados Central, pois todas as informações estão concentradas nela. Uma vantagem evidente deste procedimento é a garantia de rapidez, segurança e integridade na tomada de decisões, pois existe facilidade na obtenção dos dados (tanto dados atualizados quanto dados históricos), no entanto, a centralização das informações de todas as fábricas em uma base de dados corre o risco de gerar inconsistência e redundância dos dados, pelo fato

Tabela 1: Classificação dos clusters calçadistas e mecanismos de alocação de pedidos.

\begin{tabular}{|c|c|c|}
\hline & CARACTERÍSTICA DAS FÁBRICAS & MEGANISMO DE ALOGAÇÃO \\
\hline GLUSTERS MULTIPROCESSO & $\begin{array}{l}\text { Fábricas com todos os processos de } \\
\text { produção }\end{array}$ & $\begin{array}{l}\text { Alocação por Fragmentação de } \\
\text { pedidos }\end{array}$ \\
\hline CLUSTERS MONOPROCESSO & $\begin{array}{l}\text { Fábricas com um único processo de } \\
\text { produção }\end{array}$ & Alocação por Processo de produção \\
\hline GLUSTERS HÍBRIDOS & $\begin{array}{l}\text { São encontradas fábricas de ambas as } \\
\text { características }\end{array}$ & $\begin{array}{l}\text { Alocação Híbrida (fragmentação e } \\
\text { processo) }\end{array}$ \\
\hline
\end{tabular}


de as associadas também possuírem em suas bases de dados locais muitas das informações já contidas na Base de Dados Central. Este fato demonstra ainda mais a importância de as fábricas manterem seus dados sempre confiáveis (consistentes) por meio de um sistema de apontamento/monitoramento.

Para maior efetividade do sistema de alocação de pedidos, o agente coordenador foi implementado de modo a suportar os atributos de um Sistema de Apoio à Decisão (DSS), o que inclui a proposta de uma interface amigável, o suporte às simulações de alocação dos pedidos (Base de Modelos) e a realização de atividades de um Sistema de Planejamento e Controle da Produção.

Aplicando a estrutura de um DSS (LAUDON \& LAUDON, 1999) ao problema tratado, o agente coordenador deve incorporar em sua estrutura tecnológica uma Base de Dados capaz de sustentar todos os eventos (processos) do Industrial Cluster, uma Base de Modelos que implemente os objetivos e as restrições de um determinado negócio e dispor de interfaces amigáveis que facilitem a interação e compreensão do usuário.

\section{MODELOS}

Nesta seção, são descritos os três modelos matemáticos que fazem parte das propostas de soluções apresentadas para o problema de alocação dos pedidos em clusters calçadistas. O primeiro deles trata o mecanismo de alocação por fragmentação de pedidos. Na seqüência, as duas próximas seções trazem respectivamente os modelos matemáticos para o mecanismo de alocação por processo e para o mecanismo de alocação híbrida.

\section{Modelo Matemático para a Alocação por Fragmentação de Pedidos}

A proposta de solução para a alocação de pedidos em

Figura 1: Infra-estrutura de coordenação para um aglomerado industrial.

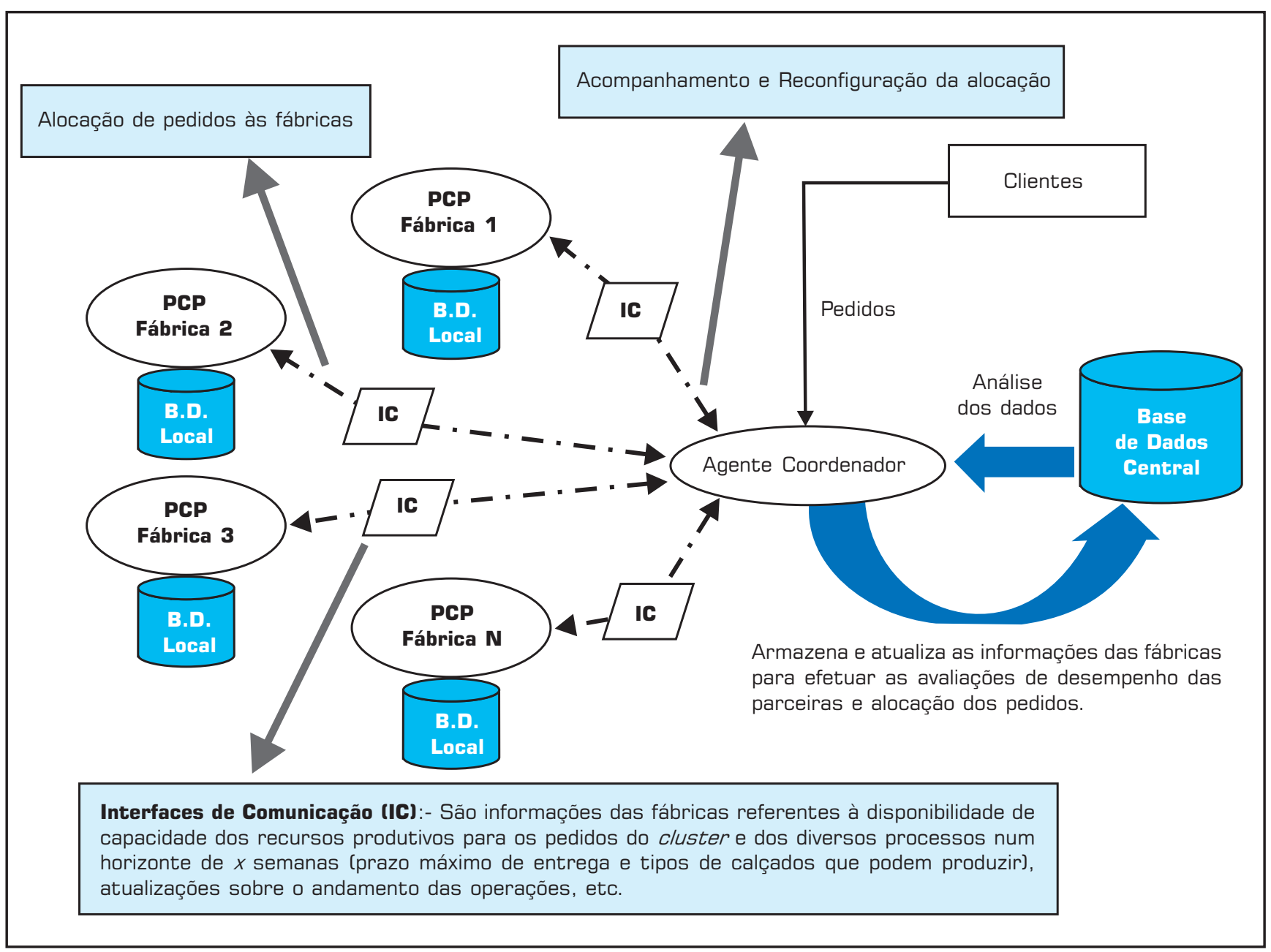


clusters multiprocesso deriva do mecanismo de alocação por fragmentação de pedidos. Tal mecanismo consiste na divisão dos pedidos em lotes de pedidos menores, ou seja, após a análise dos critérios de alocação (aptidão para produzir o lote, capacidade disponível, prazo de entrega, prioridade de recebimento, etc.), o agente coordenador fragmenta os pedidos em lotes de pedidos menores de acordo com as condições de produção das fábricas selecionadas para a alocação dos lotes.

Para o mecanismo de alocação por fragmentação de pedidos, o principal condicionante é a aptidão das fábricas em produzir os lotes de pedidos menores. Esta condição está relacionada em especial às linhas dos produtos oferecidos por cada fábrica do cluster e conseqüentemente à seqüência dos processos produtivos, de modo que, quanto mais próximas as fábricas estiverem das características do pedido fragmentado, maior é a chance delas serem selecionadas para a alocação. Uma característica importante deste mecanismo de alocação é a manutenção constante do balanceamento no chão-de-fábrica de cada membro do cluster por preservar a seqüência produtiva sempre em uma mesma fábrica.

Desta forma, desenvolveu-se um Modelo de Programação Inteira 0-1 inspirado inicialmente no Problema de Múltiplas Mochilas (MARTELLO \& TOTH, 1990). O modelo matemático proposto para cluster multiprocesso pode ser solucionado por meio de algoritmos de programação inteira 0-1, cuja função a ser otimizada compreende alocar o maior número de pares de calçados possível de um determinado subitem i' (numeração de um produto ou numeração de um item do pedido recebido) às fábricas do cluster habilitadas a produzilo, é dada por:

$$
\min \mathrm{X}_{0}=\sum_{i^{\prime}} \sum_{j} \mathrm{p}_{j} \mathrm{n}_{i^{\prime}} \mathrm{x}_{i^{\prime} j}
$$

\section{em que:}

$\mathrm{X}_{0}=$ Função Objetivo;

$i^{\prime}=$ uma numeração de um item ou produto que compõe um determinado pedido (subitem);

$j \quad$ = fábrica do Industrial Cluster

$\mathrm{p}_{j}=$ prioridade da fábrica $j$ receber subitem;

$\mathrm{n}_{i^{\prime}}=$ número de pares do subitem $i^{\prime}$;

$\mathrm{x}_{i^{\prime} j}=1$ se o subitem $i$ ' for alocado por fragmentação de pedidos à fábrica $j$;

$\mathrm{x}_{i^{\prime} j}=0$ se o subitem $i$ ' não for alocado por fragmentação de pedidos à fábrica $j$.

O modelo matemático apresentado está sujeito às seguintes restrições:

\section{Capacidade de produção:}

$\sum_{i^{\prime}} \mathrm{tc}_{i^{\prime} j} \mathrm{n}_{i^{\prime}} \mathrm{x}_{i^{\prime} j} \leq \mathrm{dc}_{j}$ para todo $j=1, . .$, (no de fábricas).

$\sum_{i^{\prime}} \operatorname{tp}_{i^{\prime} j} \mathrm{n}_{i^{\prime}} \mathrm{x}_{i^{\prime} j} \leq \mathrm{dp}_{j}$ para todo $j=1, . .$, (no de fábricas).

$\sum_{i^{\prime}} \mathrm{tm}_{i^{\prime} j} \mathrm{n}_{i^{\prime}} \mathrm{x}_{i^{\prime} j} \leq \mathrm{dm}{ }_{j}$ para todo $j=1, . .$, (no de fábricas).

em que:

tc $_{i^{\prime} j}=$ tempo (em horas) para cortar um par do subitem $i$ ' na fábrica $j$;

$\mathrm{dc}_{j}=$ disponibilidade líquida (em horas) do corte da fábrica $j$ no horizonte de planejamento;

$\operatorname{tp}_{i^{\prime} j}=$ tempo para pespontar um par do subitem $i^{\prime}$ na fábrica $j$;

$\mathrm{dp}_{j}=$ disponibilidade líquida (em horas) do pesponto da fábrica $j$ no horizonte de planejamento;

$\mathrm{tm}_{i^{\prime} j}=$ tempo para montar um par do subitem $i$ ' na fábrica $j$;

$\mathrm{dm}_{j}=$ disponibilidade líquida (em horas) da montagem da fábrica $j$ no horizonte de planejamento.

\section{Alocação do subitem $i$ ' a uma única fábrica:}

$$
\begin{aligned}
\sum_{j} \mathrm{x}_{i^{\prime} j} & =1 \text { para todo } i^{\prime} \\
\mathrm{x}_{i^{\prime} j} & =1 \text { ou } 0 \text { para todo } i^{\prime} \text { e todo } j
\end{aligned}
$$

\section{Competência:}

$$
\mathrm{x}_{i^{\prime} j} \leq \mathrm{C}_{i^{\prime} j} \text { para todo } i \text { ' e todo } j
$$

em que:

$\mathrm{C}_{i^{\prime} j}=1$ se o subitem $i$ ' pode ser produzido pela fábrica $j$;

$\mathrm{C}_{i^{\prime} j}=0$ se o subitem $i^{\prime}$ não pode ser produzido pela fábrica $j$;

Para o modelo acima apresentado, devemos citar algumas considerações:

- Um subitem corresponde a uma numeração de um item (produto) que por sua vez pertence a um determinado pedido, isto é, um subitem é composto pelo modelo YY, seu número BB (a grade da numeração dos calçados varia conforme o público) e sua quantidade de pares ZZ. A formação de um subitem é determinada pelo mecanismo de alocação por fragmentação de pedidos. A Figura 2 traz resumidamente um exemplo hipotético de um pedido recebido pelo agente coordenador. 
Por meio do exemplo ilustrado pela Figura 2, consegue-se facilmente identificar um subitem. Utilizando o produto Y1 (primeiro produto do pedido PPPPP) como referência, pode-se notar que ele tem como características a cor (CC) e entre a grade oferecida (14 até 31) foram encomendados para algumas numerações (BB) quantidades de pares específicas (ZZ) que resultaram em um total seguido de seu valor. Deste produto são criados sete subitens, isto é, para cada numeração encomendada do produto Y1 cria-se um subitem. É este subitem que será alocado à fábrica apta para sua produção.

- A prioridade $\left(\mathrm{p}_{j}\right)$ da fábrica $j$ em receber subitens é calculada pela razão:

$\mathbf{R}_{j}=\frac{\text { Quantidade de pares que a fábrica } j \text { já recebeu pelo } \text { cluster }}{\text { Número de funcionários da produção da fábrica } j}$

- Os valores de $\mathrm{R}_{j}$ são organizados em ordem crescente. A fábrica com menor $\mathrm{R}_{j}$ recebe $\mathrm{p}_{j}=1$ ou prioridade 1 (maior prioridade em receber sub-itens), o segundo menor $\mathrm{R}_{j}$ corresponderá à prioridade 2 , e assim sucessivamente. Por meio deste critério, atribuem-se valores menores $\left(\mathrm{p}_{j}\right)$ às fábricas com maior prioridade em receber subitens e assim se consegue alocar para tais fábricas quantidades maiores de pares $\left(\mathrm{n}_{i}\right.$, ) sem afetar o resultado esperado pela função objetivo, que é minimizar a somatória proposta.

- Os tempos para cortar, pespontar e montar $\left(\mathrm{tc}_{i^{\prime} j}, \mathrm{tp}_{i^{\prime} j} \mathrm{e}\right.$ tm ${ }_{i, j}$ ) um par do subitem $i$ ' na fábrica $j$ já devem estar com os seus respectivos valores divididos pelo número de funcionários de cada processo para todo $i$ ' e todo $j$. Este cálculo informa o tempo real para cortar, pespontar e montar um par do subitem $i$ '.

- O horizonte de planejamento (hp) para estabelecer a disponibilidade (em horas) da capacidade dos processos ( $\mathrm{dc}_{j}, \mathrm{dp}_{j}$ e dm ${ }_{j}$ ) será dado pelo gerente do cluster. Se o gerente escolher um hp que torne impossível a solução do modelo, ele deve aumentar o horizonte e rodar novamente o modelo. Este é um dos aspectos em que aparece a interação usuário-sistema no ambiente do DSS (Decision Support System) proposto. O cálculo da capacidade disponível de um processo é a sua capacidade total no hp menos a capacidade já destinada a alguma produção futura dentro do hp.

- O prazo de entrega de um determinado pedido é igual ao último prazo de entrega dos subitens $i$ ' pertencentes a ele.

- Caso não resulte solução ótima, verifique se existe (m) subitem (s) com quantidade maior que as capacidades das fábricas do cluster. Caso exista, particione os subitens que não satisfizeram tal condição e execute o modelo novamente.

Depois de obtida a alocação dos subitens levando em conta a capacidade disponível, é feita uma verificação se os prazos de entrega podem ser atendidos. Uma forma de minimizar o maior tempo de atraso é ordenar os subitens alocados à fábrica $j$ segundo a ordem crescente do prazo de entrega ou prazo de entrega dividido pelo número de pares (regra de seqüenciamento EDD (earliest due date)). Um detalhamento desta análise é apresentado nas considerações finais.

\section{Modelo matemático para a alocação por processo}

Este modelo matemático é uma proposta de solução para o problema de alocação dos pedidos em clusters monoprocesso. O mecanismo de alocação de pedidos

Figura 2: Pedido recebido pelo agente coordenador.

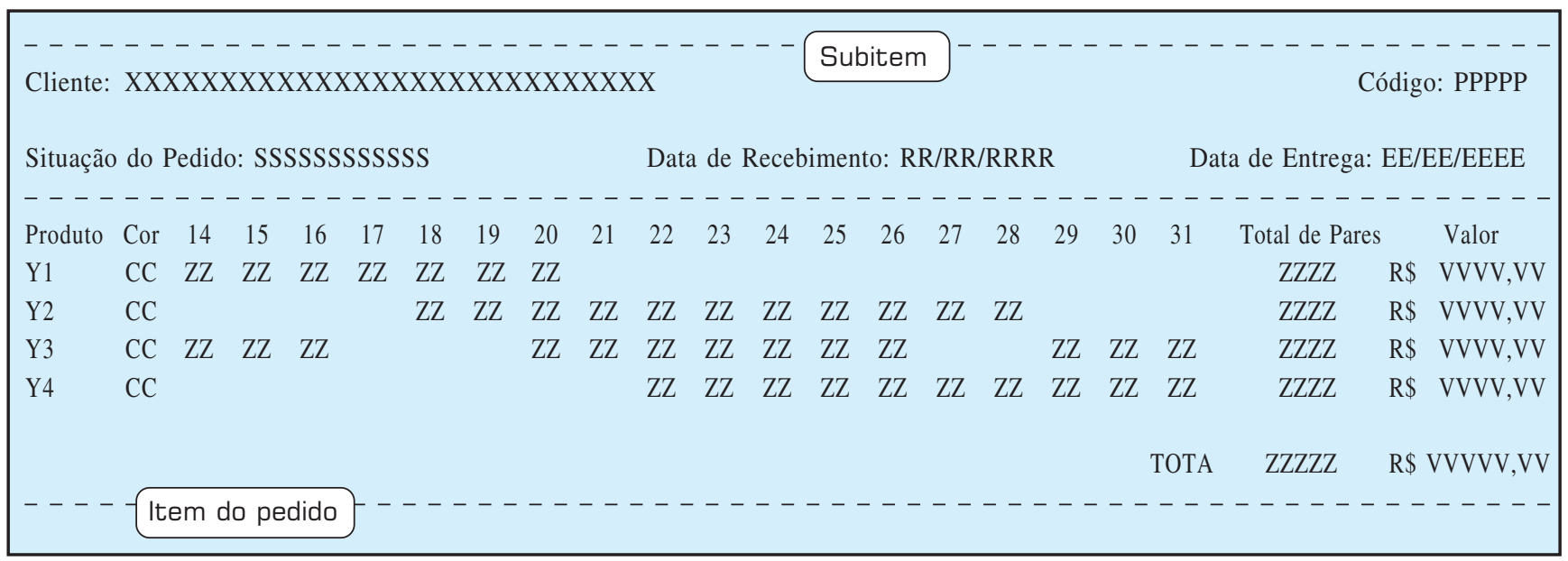


adotado é o mecanismo de alocação por processo que compreende a atribuição dos pedidos de acordo com os principais processos de fabricação dos calçados (corte, pesponto, montagem), de modo que as fábricas são selecionadas pelo coordenador conforme a aptidão pela produção do produto, a especialização do processo de produção, o prazo de término da operação e a capacidade disponível que cada uma possui em relação aos principais processos citados. Os clusters monoprocesso devem possuir no mínimo três fábricas, cada uma responsável por um dos principais processos de produção.

A função a ser otimizada neste modelo matemático consiste em alocar o maior número de pares de calçados possível de um determinado item $i$ (um produto pertencente a um pedido) às fábricas do cluster habilitadas a produzi-lo. Vale frisar que a semelhança do modelo matemático para a alocação por processo com o modelo proposto para a fragmentação de pedidos é evidente, os índices utilizados, as variáveis de decisão e as restrições seguem a mesma lógica, no entanto, as diferenças estão na utilização dos conjuntos de fábricas agrupadas por processos de produção específicos ( $\mathrm{U}_{c}, \mathrm{U}_{p}$ e $\mathrm{U}_{m}$ ) e na alocação de todas as quantidades solicitadas das respectivas numerações pertencentes a um determinado item $i$ e que por sua vez compõe um pedido (veja Figura 2).

Em relação aos conjuntos de fábricas que receberão os itens de um pedido, deve-se considerar que um item de um determinado pedido será alocado somente para uma fábrica de cada conjunto, ou seja, um item será alocado para uma fábrica do conjunto $\mathrm{U}_{c}$, cuja função é cortar o material necessário para atender tal item. Em seguida, esse mesmo item será pespontado em uma das fábricas do conjunto $\mathrm{U}_{p}$ e por fim, montado também por uma fábrica do conjunto $\mathrm{U}_{m}$. Este procedimento está de acordo com as características das fábricas dos clusters monoprocesso e acaba forçando a atribuição dos itens de um pedido sempre para três fábricas que cumprirão a sequiência de produção dos mesmos. O modelo matemático para a alocação por processo é dado abaixo:

$\min \mathrm{X}_{0}=\sum_{i} \sum_{j} \mathrm{p}_{j} \mathrm{n}_{i} \mathrm{x}_{i j}$

em que:

$\mathrm{X}_{0}=$ Função Objetivo;

$\mathrm{p}_{j}=$ prioridade da fábrica $j$ receber item;

$\mathrm{n}_{i}=$ número de pares do item $i$;

$\mathrm{x}_{i j}=1$ se o item $i$ for alocado por processo à fábrica $j$;

$\mathrm{x}_{i j}=0$ se o item $i$ não for alocado por processo à fábrica $j$.
Observação: A restrição de alocação do item $i$ às fábricas responsáveis por sua produção (corte, pesponto e montagem) garante que a variável $x_{i j}$ da função objetivo seja igual a 1 sempre para três fábricas do cluster.

O modelo matemático para a alocação por processo está sujeito às seguintes restrições:

\section{Capacidade de produção:}

$$
\begin{aligned}
& \sum_{i} \mathrm{tc}_{i j} \mathrm{n}_{i} \mathrm{x}_{i j} \leq \mathrm{dc}_{j} \text { para todo } j \in \mathrm{U}_{i} . \\
& \sum_{i} \mathrm{tp}_{i j} \mathrm{n}_{i} \mathrm{x}_{i j} \leq \mathrm{dp}_{j} \quad \text { para todo } j \in \mathrm{U}_{p} . \\
& \sum_{i} \mathrm{tm}_{i j} \mathrm{n}_{i} \mathrm{x}_{i j} \leq \mathrm{dm}{ }_{j} \text { para todo } j \in \mathrm{U}_{m} .
\end{aligned}
$$

em que:

$\mathrm{U}_{c}=$ conjunto das fábricas que só têm o processo corte;

$\mathrm{U}_{p}=$ conjunto das fábricas que só têm o processo pesponto;

$\mathrm{U}_{m}=$ conjunto das fábricas que só têm o processo montagem;

tc $_{i j}=$ tempo para cortar um par do item $i$ na fábrica $j$;

$\mathrm{dc}_{j}=$ disponibilidade (em horas) do corte da fábrica $j$ no horizonte de planejamento;

tp $_{i j}=$ tempo para pespontar um par do item $i$ na fábrica $j$;

$\mathrm{p}_{j}=$ disponibilidade (em horas) do pesponto da fábrica $j$ no horizonte de planejamento;

$\mathrm{tm}_{i j}=$ tempo para montar um par do item $i$ na fábrica $j$; $\mathrm{dm}_{j}=$ disponibilidade (em horas) da montagem da fábrica $j$ no horizonte de planejamento.

Alocação do item $i$ às três fábricas (corte, pesponto $\mathrm{e}$ montagem):

$$
\begin{aligned}
& \sum_{j \in \mathrm{U}_{c}} \mathrm{x}_{i j}=1 \quad \text { para todo } i \\
& \sum_{j \in \mathrm{U}_{p}} \mathrm{x}_{i j}=1 \quad \text { para todo } i \\
& \sum_{j \in \mathrm{U}_{m}} \mathrm{x}_{i j}=1 \quad \text { para todo } i \\
& \mathrm{x}_{i j}=1 \text { ou } 0 \text { para todo } i \text { e todo } j
\end{aligned}
$$




\section{Competência:}

$$
\begin{aligned}
& \mathrm{x}_{i j} \leq \mathrm{C}_{i j} \text { para todo } i \text { e todo } j \in \mathrm{U}_{i} . \\
& \mathrm{x}_{i j} \leq \mathrm{C}_{i j} \text { para todo } i \text { e todo } j \in \mathrm{U}_{p} . \\
& \mathrm{x}_{i j} \leq \mathrm{C}_{i j} \text { para todo } i \text { e todo } j \in \mathrm{U}_{m} .
\end{aligned}
$$

em que:

$$
\begin{aligned}
\mathrm{C}_{i j}= & 1 \text { se o item } i \text { pode ser produzido pela fábrica } j \\
& \text { em seu respectivo conjunto; }
\end{aligned}
$$

\section{Modelo Matemático para a Alocação Híbrida}

Em relação aos clusters híbridos, sugere-se a alocação híbrida como mecanismo de alocação dos pedidos que nada mais é do que a junção das soluções propostas para os clusters multiprocesso e clusters mono-processo. Para a alocação híbrida, consideramos que entre os produtos oferecidos por um cluster existem aqueles que são produzidos por fábricas que realizam todo o processo de produção, aqueles produzidos por fábricas que realizam processos de produção específicos e por fim, aqueles produtos que podem tanto ser produzidos por fábricas multiprocesso quanto por fábricas monoprocesso.

Diante disto, considera-se inicialmente para este modelo matemático a utilização de um procedimento capaz de separar, em cada pedido recebido, os itens que somente serão produzidos pelas fábricas que realizam todo o processo produtivo e que fazem parte do conjunto $\mathrm{V}$ (solução $\rightarrow$ alocação por fragmentação) e os itens que somente serão produzidos pelas fábricas que realizam processos específicos de produção e que fazem parte dos conjuntos $\mathrm{U}_{c}, \mathrm{U}_{p}$ e $\mathrm{U}_{m}$ (solução $\rightarrow$ alocação por processo).

Os itens não separados (aqueles aceitos pelos dois mecanismos de alocação) serão submetidos ao modelo matemático (abaixo) responsável pela decisão de encaminhá-los para os mecanismos de alocação por fragmentação ou processo, considerando critérios como prioridade das fábricas e balanceamento (equilíbrio) da separação dos itens restantes. O motivo pelo qual se deseja garantir tal balanceamento consiste em fazer com que os pedidos fiquem prontos o quanto antes. Seria uma solução ruim o conjunto $\mathrm{V}$ ficar sobrecarregado e os conjuntos $\mathrm{U}_{c}, \mathrm{U}_{p}$ e $\mathrm{U}_{m}$ com muita ociosidade ou viceversa. Quanto menor o volume de produção já recebido por

\begin{tabular}{|c|c|c|c|c|}
\hline$|\mathrm{R}|=$ & $\frac{\sum \mathrm{KC}_{i} \mathrm{y}_{i}}{\text { Cap. Corte V }}$ & $\frac{\mathrm{QV}}{\mathrm{QV}+\mathrm{QU}_{\mathrm{C}}}$ & $\frac{\sum_{\mathrm{KC}_{i}\left(1-\mathrm{y}_{i}\right)}}{\text { Cap. Corte } \mathrm{U}_{c}}$ & $\frac{\mathrm{QU}_{c}}{\mathrm{QV}+\mathrm{QU}_{c}}$ \\
\hline$|S|=$ & $\frac{\sum \mathrm{KP}_{i} \mathrm{y}_{i}}{\text { Cap. Pesp. V }}$ & $\frac{\mathrm{QV}}{\mathrm{QV}+\mathrm{QU}_{p}}$ & $\frac{\sum_{\mathrm{KP}_{i}\left(1-\mathrm{y}_{i}\right)} \text { Cap. Pesp. } \mathrm{U}_{p}}{{ }}$ & $\frac{\mathrm{QU}_{p}}{\mathrm{QV}+\mathrm{QU}_{p}}$ \\
\hline$|\mathrm{T}|=$ & $\frac{\sum_{\mathrm{KM}_{i} \mathrm{y}_{i}}}{\text { Cap. Mont. V }}$ & $\frac{\mathrm{QV}}{\mathrm{QV}+\mathrm{QU}_{n}}$ & $\frac{\sum_{\mathrm{KM}_{i}\left(1-\mathrm{y}_{i}\right.}}{\text { Cap. Mont. } \mathrm{U}_{m}}$ & $\frac{\mathrm{QU}_{m}}{\mathrm{QV}+\mathrm{QU}}$ \\
\hline
\end{tabular}
um conjunto de fábricas, maior a prioridade deles receberem novos itens. Assim, para levar em conta simultaneamente balanceamento e prioridade propomos o seguinte modelo matemático com a seguinte função objetivo:

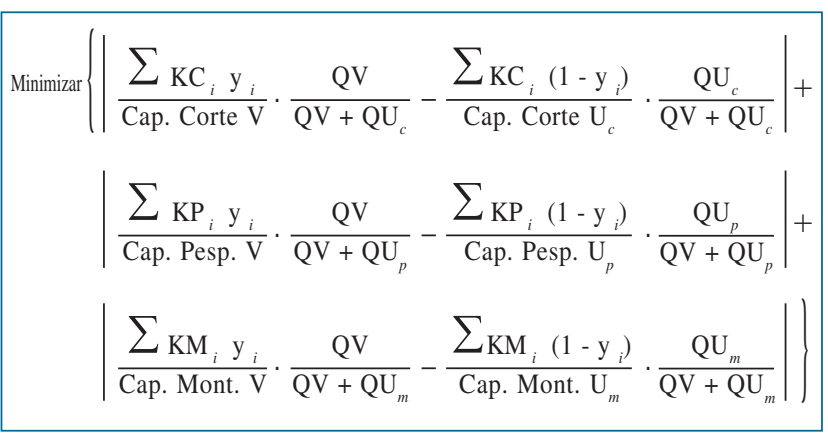

Simplificando, temos:

Portanto:

\section{Minimizar $\quad\{|R|+|S|+|T|\}$}

Para linearizar esta função objetivo, fazemos:

$$
\text { Minimizar }\{\mathrm{R}+\mathrm{S}+\mathrm{T}\}
$$

Sujeito a:

$$
\begin{aligned}
& \frac{\sum \mathrm{KC}_{i} \mathrm{y}_{i}}{\text { Cap. Corte } \mathrm{V}} \cdot \frac{\mathrm{QV}}{\mathrm{QV}+\mathrm{QU}_{c}}-\frac{\sum_{\mathrm{KC}_{i}\left(1-\mathrm{y}_{i}\right)}}{\text { Cap. Corte } \mathrm{U}_{c}} \cdot \frac{\mathrm{QU}_{c}}{\mathrm{QV}+\mathrm{QU}_{c}} \leq \mathrm{R}
\end{aligned}
$$

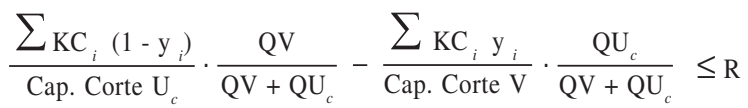

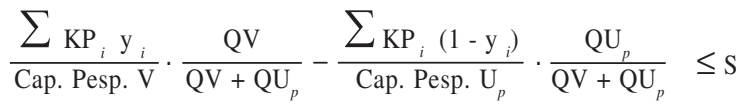

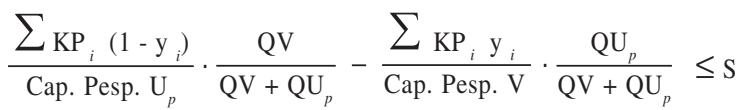

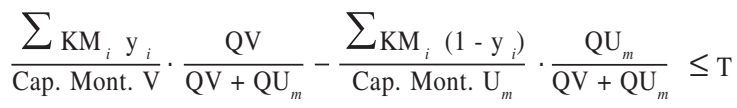

$$
\begin{aligned}
& \frac{\sum_{\mathrm{KM}_{i}\left(1-\mathrm{y}_{i}\right)}}{\text { Cap. Mont. } \mathrm{U}_{m}} \cdot \frac{\mathrm{QV}}{\mathrm{QV}+\mathrm{QU}_{m}}-\frac{\sum_{\mathrm{KM}_{i} \mathrm{y}_{i}}}{\text { Cap. Mont. } \mathrm{V}} \cdot \frac{\mathrm{QU}_{m}}{\mathrm{QV}+\mathrm{QU}_{m}} \leq \mathrm{T}
\end{aligned}
$$


Em que:

$\mathrm{QV}=$ Quantidade de pares que as fábricas do conjunto $\mathrm{V}$ já receberam.

$\mathrm{QU}_{c}=$ Quantidade de pares que as fábricas do conjunto $\mathrm{U}_{c}$ já receberam.

$\mathrm{QU}_{p}=$ Quantidade de pares que as fábricas do conjunto $\mathrm{U}_{p}^{p}$ já receberam.

$\mathrm{QU}_{m}=$ Quantidade de pares que as fábricas do conjunto $\mathrm{U}_{m}$ já receberam.

Cap. Corte $\mathrm{V}=$ Capacidade (em horas) restante do corte das fábricas $\in \mathrm{V}$.

Cap. Pesp. V = Capacidade (em horas) restante do pesponto das fábricas $\in \mathrm{V}$.

Cap. Pesp. V = Capacidade (em horas) restante da montagem das fábricas $\in \mathrm{V}$.

Cap. Corte $\mathrm{U}_{c}=$ Capacidade (em horas) restante do corte das fábricas $\in \mathrm{U}$.

Cap. Corte $U_{p}=$ Capacidade (em horas) restante do pesponto das fábricas $\in \mathrm{U}_{p}$.

Cap. Corte $\mathrm{U}_{m}=$ Capacidade (em horas) restante da montagem das fábricas $\in \mathrm{U}_{m}$.

$\mathrm{y}_{i}=1$ se o item $i$ for atribuído por fragmentação.

$\mathrm{y}_{i}=0$ se o item $i$ for atribuído por processo.

$\mathrm{KC}_{i}=$ carga em horas do corte do item $i$.

$\mathrm{KP}_{i}=$ carga em horas do pesponto do item $i$.

$\mathrm{KM}_{i}=$ carga em horas da montagem do item $i$.

Encerrado o processo de separação dos itens dos pedidos recebidos que somente serão produzidos pelas fábricas do conjunto $\mathrm{V}$ daqueles itens que somente serão produzidos pelas fábricas dos conjuntos $\mathrm{U}_{c}, \mathrm{U}_{p} \mathrm{e} \mathrm{U}_{m} \mathrm{e}$ finalizado o processo de encaminhamento dos itens híbridos (produzidos por todas as fábricas do cluster) conforme os critérios desta decisão (prioridade de recebimento e balanceamento da separação), pode-se obter a solução para o problema de alocação dos pedidos em clusters híbridos por meio da execução dos modelos matemáticos específicos aos conjuntos de fábricas, isto é, o modelo matemático para a alocação por fragmentação deve ser executado para o conjunto $\mathrm{V}$ e o modelo matemático para a alocação por processo deve ser executado para os conjuntos $\mathrm{U}_{c}, \mathrm{U}_{p} \mathrm{e} \mathrm{U}_{m}$.

\section{ESTUDO DE CASO}

O objetivo desta seção é descrever sucintamente o caso do Aglomerado Industrial Calçadista Brazon e apresentar um protótipo de sistema de informação desenvolvido para atender as necessidades relacionadas à alocação de pedidos deste cluster.

Localizado na cidade de Birigüi, Estado de São Paulo - Brasil, o cluster Brazon faz parte do Pólo Industrial responsável pela maior produção de calçados infantis do Brasil. Com uma produção diária em torno de 10.000 pares (a produção é $100 \%$ por encomenda), o cluster Brazon foi criado para que, por meio de uma produção cooperada, a parceria firmada entre suas fábricas (atualmente onze fábricas) fortaleça o grupo frente ao mercado externo, ou seja, o objetivo comum do cluster Brazon é a exportação de calçados. Este cluster tem as características de um cluster multiprocesso. Portanto, conforme explicado na seção 2 (tabela 1), o modelo a ser utilizado é o modelo 1, ou seja o modelo matemático por fragmentação de pedidos.

Uma Gerência de Exportações coordena, com a orientação da diretoria, o esforço de vendas do grupo e desempenha tarefas como: selecionar as linhas de produtos e focalizar o mercado-alvo; identificar as oportunidades de negócios de cada mercado; selecionar os melhores canais de vendas para cada mercado e obter constantemente informações atualizadas deste mercado; alocação de pedidos e acompanhamento da produção, embarque dos pedidos de acordo com o que foi combinado com o cliente; e por fim realizar o pós-venda para assim fechar a negociação.

Em termos de estabelecer prioridades ao atendimento dos pedidos externos, a Brazon atualmente inicia o processo produtivo dos pedidos por ordem de chegada, isto é, quanto antes chegar o pedido maior sua prioridade. Este critério de atendimento é na verdade definido pela fábrica responsável, que prioriza os pedidos externos para que estes não sejam atendidos com atraso e com isso não prejudique a credibilidade da Brazon.

Existe uma grande variedade de produtos $(90 \%$ de material sintético) oferecidos pela Brazon que são classificados em quatro linhas de calçados: Bebê, Infantil, Tênis e Feminino, sendo as duas últimas para os públicos infantil e adulto.

Os produtos negociados pela Brazon são unicamente destinados à exportação. Segundo a Brazon, a idéia é preservar a individualidade de cada fábrica envolvida no âmbito do mercado nacional, no entanto, existe a necessidade de estar sempre monitorando e controlando o equilíbrio entre a competição interna e a cooperação externa das fábricas envolvidas, para que a harmonia do grupo não seja enfraquecida. A manutenção do elemento confiança demonstra o grande valor que a Brazon atribui a ele, de modo que para todas as fábricas que formam tal cluster a sustentação deste grupo depende principalmente do elemento confiança. Com este propósito, reuniões periódicas são realizadas pelas fábricas envolvidas com o objetivo de analisar a situação momentânea e as perspectivas para atingir as metas futuras e como pano de fundo o fortalecimento e a união do grupo. 
Diante das preocupações destacadas pelo cluster Brazon (regras de decisão imparciais; flexibilidade e rapidez na gestão de suas operações) surgiu o protótipo SICIC (Sistema de Informação para Coordenação de um Industrial Cluster).

O SICIC é um típico Sistema de Apoio à Decisão com recursos voltados para o Planejamento e Controle da Produção. A formulação de sua Base de Modelos leva em conta as características do processo produtivo de cada fábrica que compõe o cluster Brazon. Desta forma, considerando que a Brazon possui fábricas que dominam todo o processo de produção do calçado (corte, pesponto e montagem), a base de modelos do protótipo SICIC atenderá aos critérios de alocação que compreendem a utilização do mecanismo de alocação por fragmentação de pedidos, característico de clusters multiprocesso. Diante deste ponto, é importante enfatizar que o SICIC também está apto a suportar os outros dois mecanismos de alocação (processo e híbrido) tornando-o mais completo e adaptável aos tipos de clusters definidos.

A utilização do SICIC proporciona ao agente coordenador da Brazon, ao receber um pedido, simular as condições que envolvem o processo de alocação do pedido em face das características do pedido e da situação presente (por exemplo, as cargas dos centros produtivos já alocadas, disponibilidade de matérias-primas ou leadtimes de suprimentos para obtê-las, disponibilidade de facas, possibilidades de horas extras, etc.). Ou ainda, dada a situação (pedidos e fábricas) e com base em sua experiência, o coordenador em qualquer momento pode interagir com o resultado gerado alterando algumas alocações e avaliando suas mudanças.

Pode-se entender melhor este processo observando uma de suas interfaces, a Interface Gerar Alocação (Figura 3), que disponibiliza para a alocação os pedidos em situação de pendência. Neste caso, os pedidos ficam disponibilizados em uma lista denominada Seleção de Pedidos para Alocação. Nesta lista, o gerente do cluster terá o código do pedido, sua data de entrega, a identificação do subitem formada pela junção do código do produto e da correspondente numeração do subitem e a quantidade de pares solicitada. Com essas informações, o gerente poderá selecionar os pedidos desejados utilizando o mouse (duplo clique nos subitens da coluna Alocar). Ao selecionar uma das linhas de um pedido, todos os outros subitens que correspondem ao mesmo código do pedido da linha escolhida serão selecionados para a alocação.

Outro recurso que poderá ser utilizado pelo gerente da associação nesta interface é a seleção dos subitens que serão fragmentados. O procedimento para a utilização deste recurso é semelhante à seleção dos pedidos para alocação. A diferença está na seleção um a um dos subitens com um clique duplo na coluna Frag. Não é permitido selecionar para fragmentação subitens que não estejam selecionados para a alocação.

\section{Figura 3: Interface Gerar Alocação}

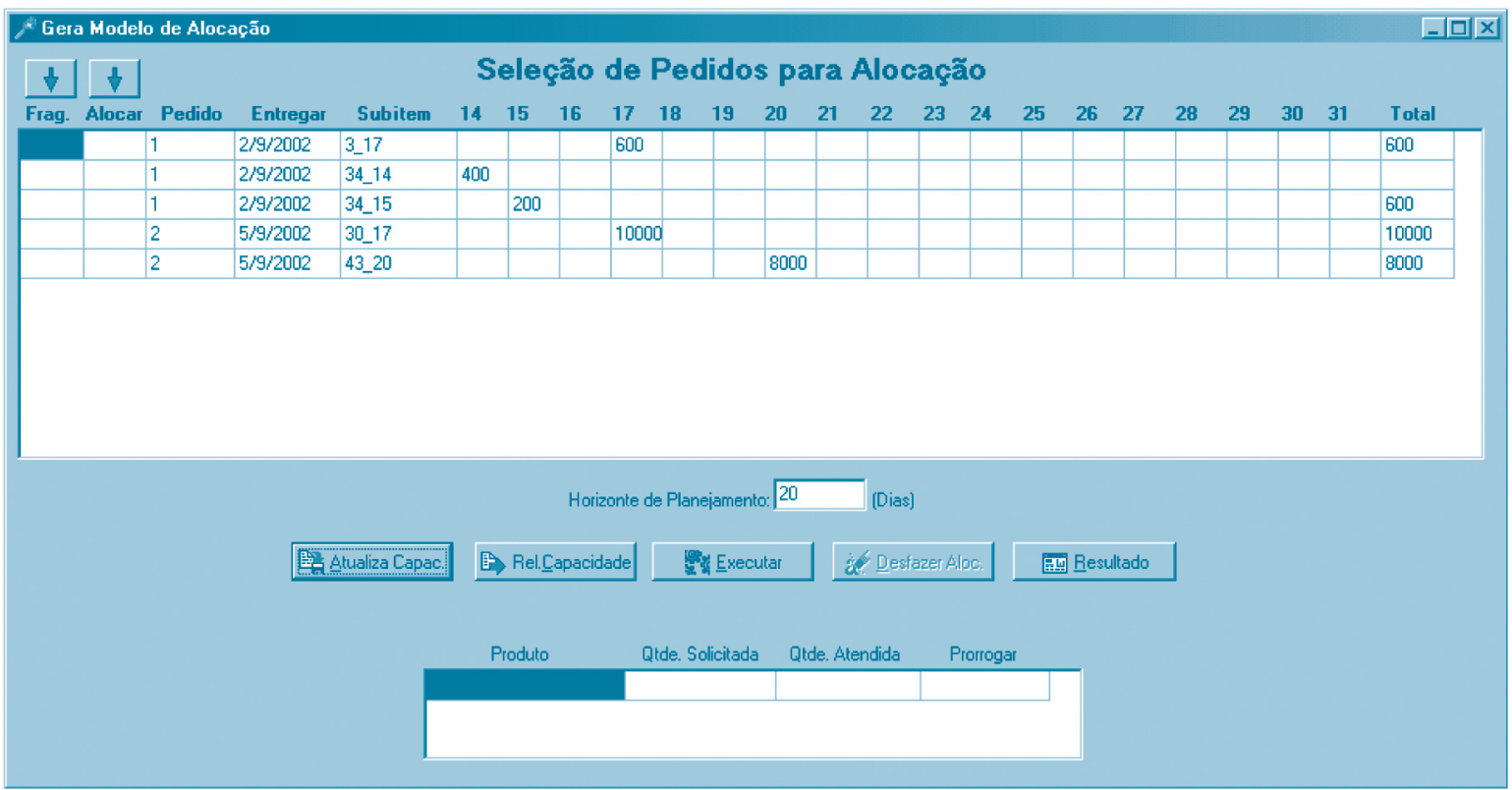


Tanto a seleção para alocação quanto a seleção para fragmentação dos subitens podem ser acionadas pelos botões posicionados acima das duas primeiras colunas da referente lista (Frag. e Alocar). Estes botões selecionam todos os subitens listados caso nenhum tenha sido selecionado e realizam a operação contrária caso todos os subitens tenham sido selecionados. Um detalhe importante da interface Gerar Alocação é que, a todo momento, o cursor aguarda a entrada do número de dias que define o horizonte de planejamento para a alocação dos subitens selecionados.

Após a realização dos procedimentos de seleção dos pedidos e a definição do horizonte de planejamento, o protótipo SICIC está apto a realizar a principal função desta interface, isto é, a alocação propriamente dita. Para que isso ocorra, o gerente deve pressionar a tecla ENTER e realizar três funções. A primeira delas é atualizar os parâmetros da capacidade disponível de cada setor produtivo. Esta função é disparada através do botão Atualiza Capac. que solicita para todas as fábricas do cluster, utilizando Interface de Comunicação, a disponibilidade dos processos produtivos dentro do horizonte de planejamento estabelecido pelo gerente. As fábricas, por sua vez, retornam os parâmetros solicitados, utilizando também Interface de Comunicação, e o SICIC atualiza sua base de dados para novas alocações.

A segunda função é solicitar um relatório de apoio (botão Rel. Capacidade) que fornece a relação entre a disponibilidade das fábricas envolvidas com o conjunto de pedidos pendentes e a quantidade de pares de cada subitem, isto é, a carga de cada setor produtivo das fábricas envolvidas. Este relatório dará a base necessária para que o gerente modifique ou mantenha a seleção dos pedidos com consciência antes de executar a alocação.

Por fim, a terceira função é efetivar a alocação dos subitens pressionando o botão Executar. Este procedimento informará se a alocação dos sub-itens selecionados foi realizada com sucesso ou não. Para o caso positivo, o gerente poderá desfazer a alocação realizada clicando sobre o botão Desfazer Aloc. e iniciar novamente todos procedimentos da interface Gerar Alocação, reconfigurando parâmetros como o horizonte de planejamento e a seleção dos pedidos, ou visualizar o resultado deste processo decisório clicando no botão Resultado.

O botão Resultado habilita uma Interface chamada Processo Decisório, que apresenta o resultado da alocação dos subitens selecionados e a situação dos subitens já alocados que estão em processo de produção, portanto, além de apresentar o processo decisório, essa interface realiza o acompanhamento da produção dos subitens já alocados.

Neste momento, o gerente toma a decisão de confirmar ou não a solução gerada. Ele decidirá tal questão a favor retornando à interface Gerar Alocação e selecionando os subitens restantes para uma nova alocação ou simplesmente fechando a interface ativa, ou ainda decidirá tal questão contra, também retornando à Interface Gerar Alocação e pressionando o botão Desfazer Aloc.

Para o caso em que a alocação não obteve uma solução viável, o gerente tem disponibilizado pela interface Gerar Alocação informações referentes ao produto, a quantidade solicitada, a quantidade atendida e a quantidade a ser prorrogada de um possível ou possíveis subitens que determinaram a inviabilidade da solução. Isto faz com que o gerente inicie novas seleções e defina novamente o horizonte de planejamento para executar a alocação dos subitens. Visando o melhor entendimento da interação existente entre o usuário (gerente) e as interfaces que envolvem de maneira geral a alocação dos subitens e o acompanhamento do processo decisório, tal interação está sintetizada no esquema definido pela Figura 4.

Com base nas características detalhadas da interface apresentada (Figura 3) e nas interações usuário-interface resumidas pela Figura 4 confirmamos o perfil do protótipo SICIC como sendo um Sistema de Apoio à Decisão (DSS). Esta confirmação deve-se principalmente ao fato de a solução alcançada ser fruto de uma interação amigável onde as informações disponibilizadas pelo SICIC subsidiam as decisões do gerente, que por sua vez coordena as iterações deste sistema de informação.

\section{$\Lambda$ implementação do agente coordenador Sistema de Apoio à Decisão (DSS).}

Vale frisar que o esquema representado pela Figura 4 só é viável se o protótipo SICIC apresentar respostas rápidas. Este requisito é perfeitamente atendido, considerando o pequeno número de novos pedidos de exportação que deverão ser alocados diariamente ou, em alguns casos, rigorosamente alocados em um prazo de uma semana.

Apesar da pequena quantidade de pedidos a serem alocados, o volume de pares dos produtos que compõem tais pedidos é grande. Mesmo assim, o modelo de programação inteira é perfeitamente viável de ser aplicado nas situações reais, portanto, não há necessidade de exaustivos testes computacionais e nem a necessidade de uma 
heurística. Situações reais envolvem um número relativamente pequeno de pedidos, já que o processo de alocação deverá ser executado logo após a entrada de um novo pedido. O motivo pelo qual tal processo é executado tão rapidamente está relacionado ao procedimento de negociação do prazo de entrega dos pedidos, ou seja, o gerente deverá informar ao seu cliente sobre a possibilidade de atender ou não seu pedido na data inicialmente definida. Um período máximo para a alocação dos pedi- dos pendentes seria uma semana, considerando para esta situação a escolha do gerente em adiar a alocação de pedidos que tenham prazos longos.

Para finalizar a seção 4, uma característica essencial responsável pelo processo de execução do modelo matemático proposto para a alocação dos pedidos da Brazon é a integração entre o SICIC e o software de Modelagem Matemática GAMS (General Algebraic Modeling System). Esta integração é estabelecida a partir do mo-

Figura 4: Interação Usuário-Interface no Processo de Alocação de Pedidos.

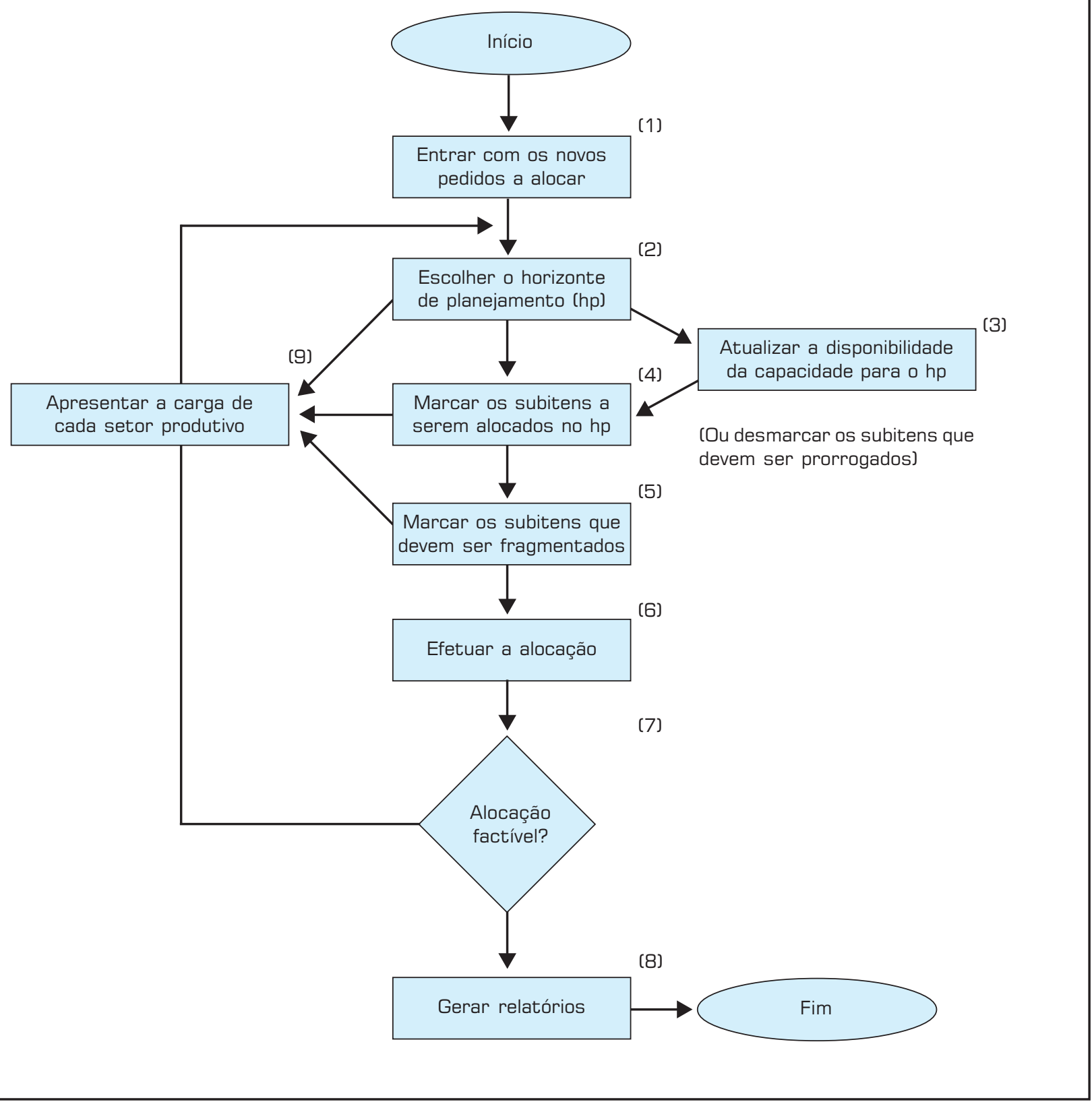


mento em que o SICIC gera um arquivo com todas as instruções referentes às restrições e à função objetivo necessárias para a alocação dos pedidos selecionados. Por sua vez, o software GAMS executa as instruções contidas no arquivo gerado e disponibiliza a solução para o SICIC, fazendo com que a solução gerada seja interpretada visualmente pelo sistema de informação da Brazon e manipulada por seu gerente. Outras ferramentas gratuitas (software livre) que implementam algoritmos de programação inteira 0-1 também podem ser utilizadas.

\section{CONSIDERACÕES FINAIS E CONCLUSÕES}

A seguir, são feitas algumas importantes considerações sobre os modelos construídos e, por último, são destacadas algumas conclusões. As considerações focam o modelo por fragmentação de pedidos, que é o modelo do estudo de caso, porém elas poderiam ser facilmente estendidas para os outros dois modelos.

O modelo é monoperíodo, porém tal período (que no caso coincide com o hp) tem duração variável. Como o ambiente é produção sob encomenda, deve-se conseguir alocar todos os pedidos já encomendados. Se o gerente escolhe um horizonte de 21 dias e ocorre infactibilidade por falta de capacidade, ele deve aumentar o horizonte, por exemplo para 28 dias, e rodar novamente o modelo, e assim por diante até encontrar o menor horizonte que gere uma solução. Esta interação usuário-sistema é uma importante característica do DSS desenvolvido. Se o ambiente fosse produção para estoque, seria mais adequado, ou até mesmo necessário, desenvolver um modelo multiperíodo.

Se o horizonte que levou à solução for maior que o prazo de um ou mais pedidos, deve haver negociação de novo prazo para certos pedidos ou pedidos que podem esperar podem ser retirados provisoriamente da alocação; nova rodada é feita até que capacidade e prazos estejam atendidos.

A outra possibilidade de ocorrer inviabilidade do modelo é haver algum pedido muito grande; porém essa possibilidade é evitada ou contornada por meio do próprio mecanismo de fragmentação de pedidos.

Depois de obtida a alocação dos subitens levando em conta a capacidade disponível, é feita uma verificação se os prazos de entrega podem ser atendidos. Uma forma de minimizar o maior tempo de atraso é ordenar os subitens alocados à fábrica $j$ segundo a ordem crescente do prazo de entrega ou prazo de entrega dividido pelo número de pares (regra de seqüenciamento EDD (earliest due date)). Se o maior tempo de atraso não for zero, deve haver negociação de novo prazo para certos pedidos, ou pedidos que podem esperar podem ser retirados provisoriamente da alocação; nova rodada é feita até que capacidade e prazos estejam atendidos. Nota-se que o sistema é um DSS e o gerente é fundamental nas negociações, e as negociações e decisões do gerente vão interferir diretamente na solução do problema. Como o gerente é parte ativa no processo decisório, obteve-se um modelo mais simples com maior potencial de aceitação para ser aplicado; geralmente, gerentes não confiam e não usam modelos complexos que eles não entendem.

A função objetivo visa alocar subitens com maior número de pares às fábricas com maior prioridade (menores valores de $p_{j}$ ). Uma possibilidade seria modelar de forma a alocar subitens com maior valor financeiro esperado (lucro esperado) às fábricas com maior prioridade (menores ganhos obtidos por elas com o aglomerado). Essa possibilidade é deixada como sugestão para pesquisas futuras, o que exigirá a necessidade de uma função contabilidade de custos apurada, algo que hoje não existe no caso estudado (Brazon).

\section{A s propostas de solução apresentadas possuem um grande potencial de implementação.}

A valorização do fator confiança por parte do cluster Brazon influenciou profundamente a definição e a utilização da prioridade de recebimento $p_{j}$ como forma de garantir a credibilidade e imparcialidade dos processos de alocação de pedidos às fábricas envolvidas. Por outro lado, em função da relação entre $p_{j}$ e $R_{j}$, vale a pena destacar como futuros trabalhos a necessidade de uma análise mais cuidadosa para tal relação, ou seja, desenvolver uma análise de sensibilidade entre estes dois parâmetros e verificar com o passar do tempo se tal relação não está alterando o desempenho do modelo matemático proposto. Esta preocupação é pertinente pelo fato de os valores atribuídos às prioridades de recebimento $p_{j}$ serem valores inteiros e serem resultantes dos valores encontrados por $R_{j}$, que são valores reais.

As propostas de solução apresentadas para aglomerados industriais calçadistas possuem um grande potencial de implementação, principalmente pelo fato de atenderem às especificações gerenciais e tecnológicas que envolvem ambientes de produção cooperada e possuírem um perfil viável às condições das pequenas e médias 
empresas, que buscam tais parcerias com o intuito de se fortalecerem perante o mercado em que atuam.

Um fator relevante para o sucesso da implementação de tais propostas é o nível de padronização das fábricas que formam um cluster (independente de sua classificação). Questões relacionadas aos padrões de qualidade das operações produtivas e matéria-prima, aos padrões dos equipamentos utilizados e aos padrões de capacitação da mão-de-obra disponível são fundamentais para a realização de uma produção cooperada (distribuída).

\section{Artigo recebido em 14/08/2004 Aprovado para publicação em 05/05/2005}

O SICIC foi testado com dados hipotéticos, porém de porte do caso Brazon, e os resultados foram promissores. O tempo de processamento utilizado pela CPU não ultrapassou em nenhum momento três segundos, considerando a elaboração do modelo matemático (restrições, variáveis, função objetivo) pelo protótipo SICIC, execução do modelo matemático (software GAMS) e a tradução e apresentação da solução obtida pelo protótipo SICIC. Em função desses resultados, um trabalho de médio prazo para a implantação efetiva do SICIC na Brazon está para ser iniciado.

\section{- Referências Bibliográficas}

AMATO NETO, J.; Redes de cooperação produtiva: antecedentes, panorama atual e contribuições para um política industrial, Tese de livre docência apresentada ao Departamento de Engenharia de Produção da Escola Politécnica da Universidade de São Paulo, 1999.

ANDRADE, J.E.P. \& CORRÊA, A.R. Panorama da Indústria Mundial de Calçados, com ênfase na América Latina, BNDES Setorial, Rio de Janeiro, $\mathrm{n}$. 13 , p. 95-126, mar. 2001

AZEVEDO, A.L. \& SOUSA, J.P. A component-based approach to support order planning in a distributed manufacturing enterprise. Journal of Materials Processing Technology, v.107, p. 431-438, 2000.

CAMAGNI, R. Local milieu, uncertainty and innovation networks: towards a new dynamic theory of economic space. In: Roberto Camagni (Ed.) Innovation Networks (London: Belhaven Press), p. 121-144, 1991.

CONTADOR, J.C. \& CONTADOR, S.L. Programação e Controle da Produção para a indústria intermitente. In: CONTADOR, S. C. (Coordenador): Gestão de Operações. São Paulo: Editora Edgar Blucher Ltda., 1997.

CORRÊA, H. \& GIANESI, I. Sistemas de Planejamento e Controle da Produção. In: CONTADOR, S. C. (Coordenador): Gestão de Operações. São PauloEditora Edgar Blucher Ltda. 1997.
COUTINHO, L.G.; FERRAZ, J.C. Parte III - Dimensão estrutural da competitividade. In: COUTINHO, L.G.; FERRAZ, J.C. Estudo da competitividade da indústria brasileira. Campinas: Papirus, 1994.

DAY, M.; BURNETT, J.; FORRESTER, P.; HASSARD, J. Britain's last industrial district? A case study of ceramics production, International Journal of Production Economics, 2000.

FERNANDES, F.C.F. \& MATTIUZZO, M. P. Diagnóstico e Avaliação de uma Pequena Empresa da Indústria Calçadista. Revista Tecnicouro, v. 8 , n. 4, p. 50-61, julho/agosto 1986.

KOVÁCS, G.L. \& MEZGÁR, I.A Distributed Planning Network for Manufacturing Systems Management, Proceedings IEEE International Conference on Robotics and Automation, v. 2, p. 1787-1792, 1998.

LAUDON, K.C. \& LAUDON, J.P. Sistemas de Informação, $4^{\circ}$ Edição com Internet, Editora LTC - Livros Técnicos e Científicos S.A., 1999.

LECOMPTE, T.; DESCHAMPS, J.C.; BOURRIERES, J. P. A data model for generalized scheduling for virtual enterprise, Production Planning and Control, v. 11, n. 4, p. 343-348, 2000.

MARTELlO, S. \& TOTH, P. Knapsack Problems - algorithms and computer implementations. Wiley, Chichester (England), 1990.
MCCORMICK, D. African Enterprise Clusters and Industrialization: Theory and Reality. World Development, v. 27, n. 9, p. 15311551, 1999.

MEZGÁR, I.; KOVÁCS, G.L. PAGANELLI, P. Co-operative production planning for small and medium-sized enterprise. International Journal of Production Economics, v. 64, p. 37-48, 2000.

PORTER, M.E. The Adam Smith address: location, clusters, and the "new" microeconomics of competition, Business Economics, v. 33, n. 1, 1998.

PORTER, $\mathrm{M}$. The Competitive Advantage of Nations. New York: The Free Press, 1990.

RABELLOTTI, R. Industrial District in Mexico - the case of the footwear industry. Small Enterprise Development, v. 4, n. 3, 1993.

RABELLOTTI, R. Is There an "Indus trial District Model"? Footwear Districts in Italy and Mexico Compared", World Development, v. 23, n. 1 , p. $29-41,1995$

RABELLOTTI, R. Recovery of a Mexican Cluster: Devaluation Bonanza or Collective Efficiency?" World Development, v.27, n. 9, p. 15711585, 1999.

RICHARDS, H.D.; DUDENHAUSEN, H. M.; MAKATSORIS, C.; RIDDER, L. Flow of orders through a virtual enterprise - their proactive planning and scheduling, and reactive control. Manufacturing Systems, August, 1997.
SANTOS, S.A.; PEREIRA, H.J.; ABRAHÃO FRANÇA, S.E. Cooperação entre as micros e pequenas empresas. SEBRAE/SP, 1994.

SCHMITZ, H. On the clustering of small firms. IDS Bulletin, v. 23, n. 3 , p. 64-69, 1992.

SCHMITZ, H. Small Shoemakers and Fordist Giants: Tale of a Supercluster", World Development, Vol.23, No.1, pp. 9-28, 1995.

SCHMITZ, H.; Global Competition and Local Cooperation: Success and Failure in the Sinos Valley, Brazil, 1999.

SIKORA, R. \& SHAW, M.J. A MultiAgent Framework for the Coordination and Integration of Information Systems. Management Science, v. 44, n. 11, Part 2 of 2, 1998

SOARES, A.L.; AZEVEDO, A.L.; SOUSA, J.P. Distributed planning and control systems for the virtual enterprise: organizational requirements and development life-cycle. Journal of Intelligent Manufacturing, v. 11, p. 253$270,2000$.

VOLLMANN, T.E.; BERRY, W.L.; WHYBARK, D.C. Manufacturing Planning and Control Systems, Irwin/ McGraw-Hill, 1997.

ZHOU, Q. \& BESANT, C. B. Information Management in Production Planning for a Virtual Enterprise, International Journal of Production Research, v. 37, n. 1, p. 207-218, 1999. 
- Agradecimentos

Os autores agradecem a Fundação de Amparo à Pesquisa do Estado de São Paulo (FAPESP) pelo apoio, o aglomerado industrial Brazon pela colaboração e os referees anônimos pelas valiosas sugestões para a melhoria deste artigo.

- Sobre os autores

Gustavo Beltran de Souza

Doutorando do Depto. de Eng. Produção - Univ. Federal de São Carlos - UFSCar

\section{Flávio César Faria Fernandes}

Prof. Dr. do Depto. de Eng. Produção - Univ. Federal de São Carlos - UFSCar

Fone: 0xx16-3351.8237 ramal 214; Fax: 0xx16-3351.8240

Endereço para correspondência: rua Dona Alexandrina, 1904 - 13.560-290 São Carlos-SP

E-mail: dfcf@power.ufscar.br 